\title{
To Decide to Ride and Bide: Information Processing Among Bus Riders
}

May 2009

\author{
Nicholas C. Holtkamp \\ Adviser: Dr. Bruce A. Weinberg
}

\begin{abstract}
:
This paper develops a model and uses data from a natural setting to test the hypothesis that people use readily available information in real-life settings. We model a traveler, who arrives at a bus stop and is faced with the choice of walking to her destination, or waiting for and then riding the bus. We show that optimal decision making implies that the traveler is more likely to wait at the bus stop the more people she sees already waiting at the bus stop. Our work differs from previous work by developing a formal game theoretic model and relatively simple testable implications. Data taken from observing travelers' behavior on The Ohio State University's Columbus campus then support the model.
\end{abstract}




\section{Introduction}

It is commonly assumed that people use the knowledge available to them to make more informed decisions. Our paper tests that assumption in the context of a bus stop. We examine whether travelers arriving at a bus stop use readily available information to minimize their travel time. We test the hypothesis that the probability that a traveler waits at a bus stop increases as the number of travelers already waiting at the bus stop also increases.

Research in the field of herd behavior suggests that people heavily weight others' decisions to make their own decisions. For example, some voters are more likely to vote for a candidate if they know that others will vote for that same candidate (Cukierman 1991). Banerjee presents a model in which people make decisions based on others' decisions rather than their own individual information. When others make decisions in the same manner, an information cascade may ensue in which all individuals act in the same manner (Banerjee 1992). Our work is similar to research in herd behavior in that we examine travelers' behavior to discover how travelers' decisions affect other travelers' decisions. In the tradition of experiments set in natural environments (Levitt and List 2008), we record data on travelers' behavior at a bus stop to test our model's predictions.

Previous research has examined how travelers use readily available information at a bus stop. McCord, Mishalani and Wirtz surveyed travelers' on their perceptions of the amount of time they waited for the bus (McCord, Mishalani and Wirtz 2006). They found that travelers perceive their wait time to be longer than their actual wait time. Consistent with this finding is that real-time information on bus arrival times reduces the waiting time and travel time of travelers (Ridho and Sumi 2009). Our paper differs from other work in that we look at a traveler deciding to wait for the bus or walk directly to her destination at a bus stop where the quickest 
method to reach her destination depends on how soon the bus will arrive. The situation posited in our study usually only occurs in a setting where destinations are close to bus stops, for example urban or campus areas.

We used theoretical and empirical methods to develop and test our hypothesis. A game theoretic model predicts travelers' wait rates at a bus stop. We examine how travelers' wait rates change based on the number of travelers waiting. In addition, we explore the effects of how often the bus arrives, how long it takes travel to the destination, and the number of travelers who have arrived at the bus stop regardless of whether they wait or walk.

Our model's predictions are then compared to empirical data taken at a bus stop on The Ohio State University's Columbus campus. Our data confirms our model's prediction, the probability that a traveler waits increases as the number of travelers seen already waiting increases, and suggests that travelers use readily available information to minimize their travel time.

\section{Model}

\section{The Framework}

Our model assumes that travelers arrive on foot at a bus stop with the intent to reach their destination. Only one bus route that arrives every $B$ minutes, called headway $B$, services the bus stop. Each traveler is assumed to be identical except for the order that they arrive at the bus stop. Upon arrival, each traveler only has two choices: to wait for the next bus to arrive, or to walk directly to her destination. The traveler does not know when the next bus will arrive; she can only predict the arrival time based on the information available to her: the headway $B$, the amount of travelers she sees waiting at the bus stop upon her arrival, and the total number of travelers who would have arrived at the bus stop over the entire headway. Travelers do not see 
that a bus is about to arrive or depart and do not prefer to wait or walk for any reason other than the amount of time it takes for them to reach their destination.

The traveler's first choice, to not wait at the bus stop and walk directly to her destination, would take her time $T_{F}$ to reach her destination. If she instead decides to wait for the bus, the time it will take her to reach her destination is the sum of the time she will wait at the bus stop, $T_{W}$, the time it will take her to ride the bus, $T_{R}$, and the time it will take her to walk from where she exits the bus to her destination, $T_{D}$. It is assumed that $T_{F}, T_{R}$ and $T_{D}$ are constant and known by the traveler.

It is assumed that the traveler is risk neutral. Therefore, the traveler is indifferent upon arrival between waiting and walking if she knows that her two choices will take the same amount of time. Furthermore, if she is indifferent, the time it takes her to walk directly to her destination compared to the time it takes her to ride the bus and walk the remaining distance to her destination must be the same amount of time she expects to wait until the next bus arrives.

$$
\begin{gathered}
T_{F}=T_{W}+T_{R E}+T_{D} \\
T_{E}=T_{F}-T_{R}-T_{D} \\
T_{E}=T_{W}
\end{gathered}
$$

Let $n$ denote the total number of travelers who will arrive over the entire bus headway $B$. Let $m$ denote the number of travelers seen waiting. For the traveler to predict her expected wait time until the next bus arrives, she not only takes into account $B$, but also $m$ and $n$. Therefore, the traveler's expected waiting time was found combinations of $m$ and $n$. 
Take the example $n=2$ and $m=1$. The two travelers are $Y$ and $Z$. When $Z$ arrives and sees one traveler already waiting, $Z$ knows that there is only one possibility: $Y$ arrived first and waited.

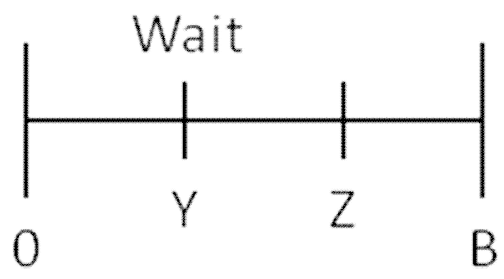

$Z$ 's expected wait time is:

$$
T_{W}=(E[\text { Wait Time I } Y \text { Arrived Before } Z)
$$

Therefore, for $Z$ to be indifferent:

$$
T_{E}=(E[\text { Wait Time I } Y \text { Arrived Before } Z)
$$

If $m=0$ instead, then $Z$ arrives and sees no one waiting. $Z$ then knows there are two possibilities: $Z$ arrived before $Y$, or $Y$ arrived before $Z$ and $Y$ walked.
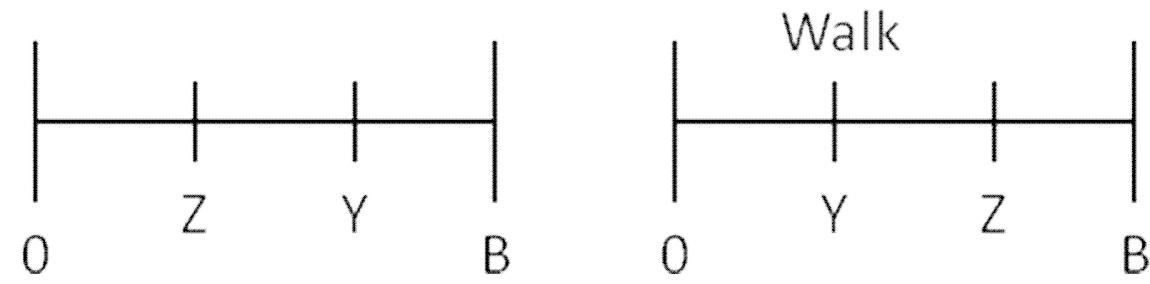

Let $W$ be the probability that $Z$ arrived before $Y$. Therefore, $1-W$ is the probability that $Y$ arrived before $Z$ and $Y$ walked. For $Z$ to be indifferent:

$T_{E}=\frac{\text { (E[Wait Time I } Z \text { Arrived Before } Y]) W+(E[\text { Wait Time I } Y \text { Arrived Before } Z])(1-W)}{W+(1-W)}$

With the framework of our model established, we then found values for the expected wait times and probabilities shown above for different values of $n$.

\section{Solving the Model}


To find values for the expected wait time given an arrival order and the probability of that arrival order, we found the expected wait times for all the combinations that travelers could have arrived under a certain $n$. For $n=2$, the only two arrival combinations are $Z$ arrived before $Y$ or $Y$ arrived before $Z$.

To find $Z$ 's expected wait time until the next bus arrives given that $Y$ arrived before $Z$, we first found Z's expected time since the last bus left:

$$
\frac{\int_{0}^{B}\left(\int_{T_{Y}}^{B} \frac{T_{Z}}{B} \mathrm{~d} T_{Z}\right) \frac{1}{B} \mathrm{~d} T_{Y}}{\int_{0}^{B}\left(\int_{T Y}^{B} \frac{1}{B} \mathrm{~d} T_{Z}\right) \frac{1}{B} \mathrm{~d} T_{Y}}=\frac{\frac{B}{3}}{\frac{1}{2}}=\frac{2 B}{3}
$$

Given that $Y$ arrived before $Z, 2 B / 3$ minutes would have elapsed between the last bus's departure and Z's arrival. Therefore, Z's expected wait time until the next bus arrives is $B / 3$ minutes. Also note that the probability that $Y$ arrived before $Z$ is $1 / 2$. We repeated this same process given that $Z$ arrived before $Y$. We found $Z$ 's expected wait time until the next bus arrives to be $2 B / 3$ minutes, and the probability that $Z$ arrived before $Y$ to be $1 / 2$.

Having found $Z$ 's expected wait times for the different arrival orders, we used the indifference relationship presented above to solve for $n=2$ and $m=1$ and $n=2$ and $m=0$. Let $\sigma_{i}$ denote the chance that a traveler walks having seen $i$ travelers waiting. In addition, let $S=$ $T_{E} / B . \mathrm{S}$ is the comparison between the extra time it takes to directly walk to the destination and the bus headway. $0 \leq S \leq 1$ must be true for the traveler to be indifferent. If $S$ is greater than 1 , then the traveler knows that it will be faster to ride the bus because the amount of extra time it takes to walk is greater than the bus headway, which is the maximum waiting time. If $S$ is less than 0 , then the traveler knows that it will be faster to walk because the amount of extra time it takes to walk will always be less than the time to wait for the bus. 
For $n=2$ and $m=1$ :

$$
\begin{gathered}
T_{E}=B-\frac{\left(\frac{2 \mathrm{~B}}{3}\right)\left(\frac{1}{2}\right)\left(1-\sigma_{0}\right)}{\left(\frac{1}{2}\right)\left(1-\sigma_{0}\right)} \\
S=1-\frac{2}{3}=\frac{1}{3}
\end{gathered}
$$

Therefore, for $Z$ to be indifferent between waiting and walking, the extra time it takes to walk must be $1 / 3$ of the bus headway.

For $n=2$ and $m=0$ :

$$
\begin{gathered}
T_{E}=B-\frac{\left(\frac{B}{3}\right)\left(\frac{1}{2}\right)+\left(\frac{2 B}{3}\right)\left(\frac{1}{2}\right) \sigma_{0}}{\frac{1}{2}+\left(\frac{1}{2}\right) \sigma_{0}} \\
\sigma_{0}=\frac{\mathrm{s}-\frac{2}{3}}{\frac{1}{3}-\mathrm{s}}
\end{gathered}
$$

If $n=2, \sigma_{0}$ is the rate that $Y$ walks seeing no one waiting that makes $Z$ indifferent between waiting and walking. If $0 \leq S<1$ and $S$ increases to not more than 1 (as explained above), then the extra time it takes to walk and the bus headway converge. As they do so, for $Z$ to remain indifferent, her expected waiting time must increase. Intuitively, Z's expected wait time increases as it becomes more likely that she arrived first rather than second; and the lower the rate that $\mathrm{Y}$ walks is, the more likely it is that $\mathrm{Z}$ arrived first if she arrives and sees on one waiting. Therefore, for $Z$ to remain indifferent as $S$ increases, the chance that $Y$ walks, $\sigma_{0}$, must decrease.

\section{When $n \geq 3$}

Extra complexity is introduced when $n=3$ as there are two different walk rates: $\sigma_{0}$, and the rate that a traveler walks seeing one person waiting, $\sigma_{1}$. To find $\sigma_{0}$ and $\sigma_{l}$ under $n=3$, we 
found $Z$ 's expected wait times for each arrival order for $n=3$. We then substituted those values into the indifference equation using the same methods we used for $n=2$. Because $\sigma_{0}$ and $\sigma_{l}$ are interdependent, we first solved for $\sigma_{0}$ to solve for $\sigma_{l}$. For $n=3$ and $m=0$ :

$$
\sigma_{0}^{2}\left(\frac{1}{4}-s\right)+\sigma_{0}\left(\frac{2}{4}-s\right)+\left(\frac{3}{4}-s\right)=0
$$

Here $\sigma_{0}$ is the rate that a traveler who arrives before $\mathrm{Z}$ and sees no one waiting walks to make $\mathrm{Z}$ indifferent. Whereas for $n=2$ there was only one solution for $\sigma_{0}$, if $n=3$, there are two solutions for $\sigma_{0}$ in the form of two roots. For $n=3$ and $m=1$ :

$$
\sigma_{1}=\frac{-\sigma_{0}\left(\frac{1}{4}-s\right)-\left(\frac{2}{4}-s\right)}{\frac{1}{4}-s}
$$

$\sigma_{l}$ is the rate that a traveler who arrives before $\mathrm{Z}$ and sees one person waiting walks to make $\mathrm{Z}$ indifferent.

The generalized equation for any $S, n, m$, and $\sigma_{0}, \sigma_{1}, \sigma_{2} \ldots \sigma_{m}$, to make $\mathrm{Z}$ indifferent as to waiting for the bus or walking to her destination is:

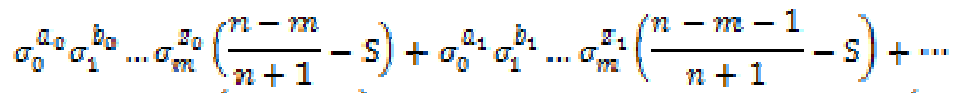

$$
\begin{aligned}
& +\sigma_{0}^{a_{n-m-1}} \sigma_{1}^{b_{n-m-1}} \ldots \sigma_{m}^{B_{n-m-1}}\left(\frac{2}{n+1}-s\right)+\sigma_{0}^{a_{n-m n-1}} \sigma_{1}^{b_{n-m-1}} \ldots \sigma_{m}^{B_{n-m-1}}\left(\frac{1}{n+1}-s\right)=0
\end{aligned}
$$

For all unique combinations of integers $\geq 0$ where $a_{\beta}+b_{\beta}+\ldots+z_{\beta}=\beta$

The appendix provides an explanation of the generalized equation.

\section{Predictions}

We only used three cases, $n=2 m=0, n=3 m=0$ and $n=3 m=1$, to find our model's predictions because while cases greater than $n=3$ are mathematically more complicated, they are innately a repetition of the same pattern found in $n=3$. In choosing values to input for $\sigma_{i}$, we 
only used the probabilities between 0 and 1 because of their application to a natural setting. Any probability equal to or less than 0 was assumed to be 0 (the traveler always waits), and any probability above or equal to 1 was assumed to be 1 (the traveler always walks). See Figure 1 for the results. Note that Figure 1 does not show the probability that travelers walk, $\sigma_{i}$, but rather the probability that travelers wait, $1-\sigma_{i}$.

Our model presents three predictions. In Figure 1, given the same $S$, the probability that travelers wait for $n=3 m=1$ is greater than the probability that travelers wait for $n=3 m=0$. Therefore, the probability that travelers wait increases as the number of travelers seen waiting increases. Intuitively, if a traveler sees that someone else is already waiting, she knows that someone else has arrived first. Therefore, she expects a shorter waiting time and will wait more often. In the comparison between $n=3 m=0$ and $n=3 m=1$ in Figure 1, when a traveler sees one person waiting, she will always wait.

Second, holding $S$ and $m$ constant, the probability that travelers wait decreases as the total number of travelers increases. Our prediction is rational in a natural setting. Holding the probability that travelers wait constant, the number of travelers that wait rises as the total number of travelers rises. Therefore, holding the number of travelers that wait constant, the probability that travelers wait decreases as the total number of travelers increases.

Third, the probability that travelers wait increases as $S$ increases. The intuitive reasoning is covered in the explanation of the solution for $\sigma_{0}$ under $n=2 m=0$ in section II.2.

\section{Data}

The researcher recorded data at the bus stop in front of University Hall, 230 N. Oval Mall, Columbus, OH, 43210, on March $6^{\text {th }}, 10^{\text {th }}, 11^{\text {th }}$ and $16^{\text {th }}, 2009$, and April $2^{\text {nd }}$ and $9^{\text {th }}, 2009$. The University Hall bus stop was the best choice out of a number of bus stops on The Ohio State 
University's Columbus campus. It was located in the center of campus and within reasonable walking distance of student housing. It serviced only two bus routes. Finally, the sign at the bus stop displayed only the headway, not the timetable, so it was more difficult for travelers to know when the next bus would arrive.

Sitting across the street so as to not affect travelers' behavior, the researcher recorded the times people waited, left (having already waited), and walked by the bus stop, and the times buses arrived and departed. The researcher did not take data when there were too many people to accurately record. People who would have seen the bus about to arrive or having just left were recorded but not included in the data used for our analysis. The researcher also recorded the temperature. Data was only taken in clear conditions. The lowest temperature reached was $39^{\circ} \mathrm{F}$ and the highest was $71^{\circ}$.

\section{Results}

Before comparing the model's predictions to the data, it's important to point out significant differences between the model's assumptions and the data's environment. While the model assumes that there is one bus route, there were two bus routes that serviced the University Hall bus stop. Neither of those bus routes consistently arrived at their promised times like the model assumes the travelers expect. The model also assumes that all travelers are identical except for the order that they arrive. Obviously, the persons arriving at the University Hall bus stop were not exactly identical. And while each person does have some preference between waiting or walking, the model does not account for any preferences. The robustness of our tests, though, seem to indicate that the differences between our model and our data should not interfere with testing our hypothesis. 
Table 1 shows the rate that travelers waited regressed on the number of people waiting, the rate that people arrived at the bus stop regardless of whether they wait or keep walking, and the temperature using Ordinary Least Squares Method. The data confirms our model's first prediction that the probability that people wait at the bus stop increases as more travelers are seen waiting at the bus stop. There is a very significant relationship between the rate that people waited and the number of people waiting. For every extra person waiting, a person waits approximately $1.5 \%$ more often (see Figure 2).

Not every person who passed the University Hall bus stop made the decision to wait or walk. To find how many of those who arrived at the bus stop made the decision to wait or walk, we looked at travelers' behavior when they would have seen the bus about to arrive. When a traveler who is making the decision to wait or walk sees the bus about to arrive, she will always wait. We found that one out of five travelers waited when they saw the bus was about to arrive. Applied to the entire data, about one out of five travelers actually made the decision to wait or walk. Therefore, travelers' rate of waiting did not increase by $1.5 \%$ for every extra person they saw waiting because that includes those who arrived at the bus stop but were simply passing by. Instead, of those making the decision to wait or walk, the rate of waiting increased by $7.5 \%$ for every extra person seen waiting.

While our work focused on testing our hypothesis, addressed by our model's first prediction, the data obtained for $n$ and the temperature reinforced the robustness of our findings. The relationship between the rate that people waited and the number of people waiting remained significant while $n$ and the temperature were not significant. Either our crude measurement of $n$, as the average number of travelers that arrived per minute over the bus headway, or travelers not taking into account $n$ when deciding to wait or walk, may explain why our model's second 
prediction was not confirmed. Regarding the temperature results, harsher weather conditions than the researcher tested may have had a more significant effect on the rate that people wait. A study of harsher weather conditions may also have implications for the usefulness of shelters at bus stops.

Finally, the researcher did not test for travelers' destinations, so the model's third prediction, while it intuitively makes sense, was not tested. Future work may examine the second and third predictions more carefully.

\section{Conclusion}

Our research confirms that travelers do use readily available data in a natural setting. Such decision making likely improves efficiency. In particular, we find that the wait rate increases as the number waiting increases. Our work implies that the more accurate a travelers' information is, the better decision the traveler will make. Displays that show the time until the next bus arrives have been installed at some bus stop on The Ohio State University's campus. A display replaces the need for a traveler to predict her expected wait time based on the number of people already waiting. Future work may test whether travelers more effectively minimize the time to reach their destination when they see a display rather than only using the number of travelers waiting. With a display installed, researchers may also examine whether the number of travelers waiting has any effect on the rate that travelers wait.

Our work did not conclusively explain how travelers use the total number of travelers to make their decision to wait or walk. Future work may find a better measure for a travelers' perception of the number of travelers who arrive over the entire bus headway to test our model's second prediction. To test the model's third prediction, researchers must find a setting where travelers' destinations and their methods to reach their destinations are known by the researcher. 
For example, a researcher may examine travelers' behavior on The Ohio State University's Columbus campus on a day when the football team is playing at home. On a home game-day, it may be safely assumed that almost every travelers' destination is the football stadium. 


\section{References}

Banerjee, Abhijit V., "A Simple Model of Herd Behavior." The Quarterly Journal of Economics 107 (No. 3 1992), 797-817.

Cukierman, Alex, "Asymmetric Information and the Electoral Momentum of Public Opinion Polls.” Public Choice 70 (No. 2 1991), 181-213.

Levitt, Steven D., and John A. List, "Field Experiments in Economics: The Past, The Present, and the Future." NBER Working Paper No. 14356, September 2008.

McCord, Mark M., Rabi G. Mishalani and John Wirtz, "Passenger Wait Time Perceptions at Bus Stops: Empirical Results and Impact on Evaluating Real-Time Bus Arrival Information." Journal of Public Transportation 9 (No.2 2006), 89-106.

Ridho, Muhammad and Tomonori Sumi, "The Effects of Accessing Real-Time Bus Arrival Information Via Mobile Phone on the Travel Time Dispersion of Transit Passengers." International Journal of Environment and Sustainable Development 8 (Number 3-4 2009), 351-364. 
Figure 1: Equilibrium Probabilities of Waiting for Travelers




Figure 2: Probabilities that Travelers Waited at Campus Bus Stop

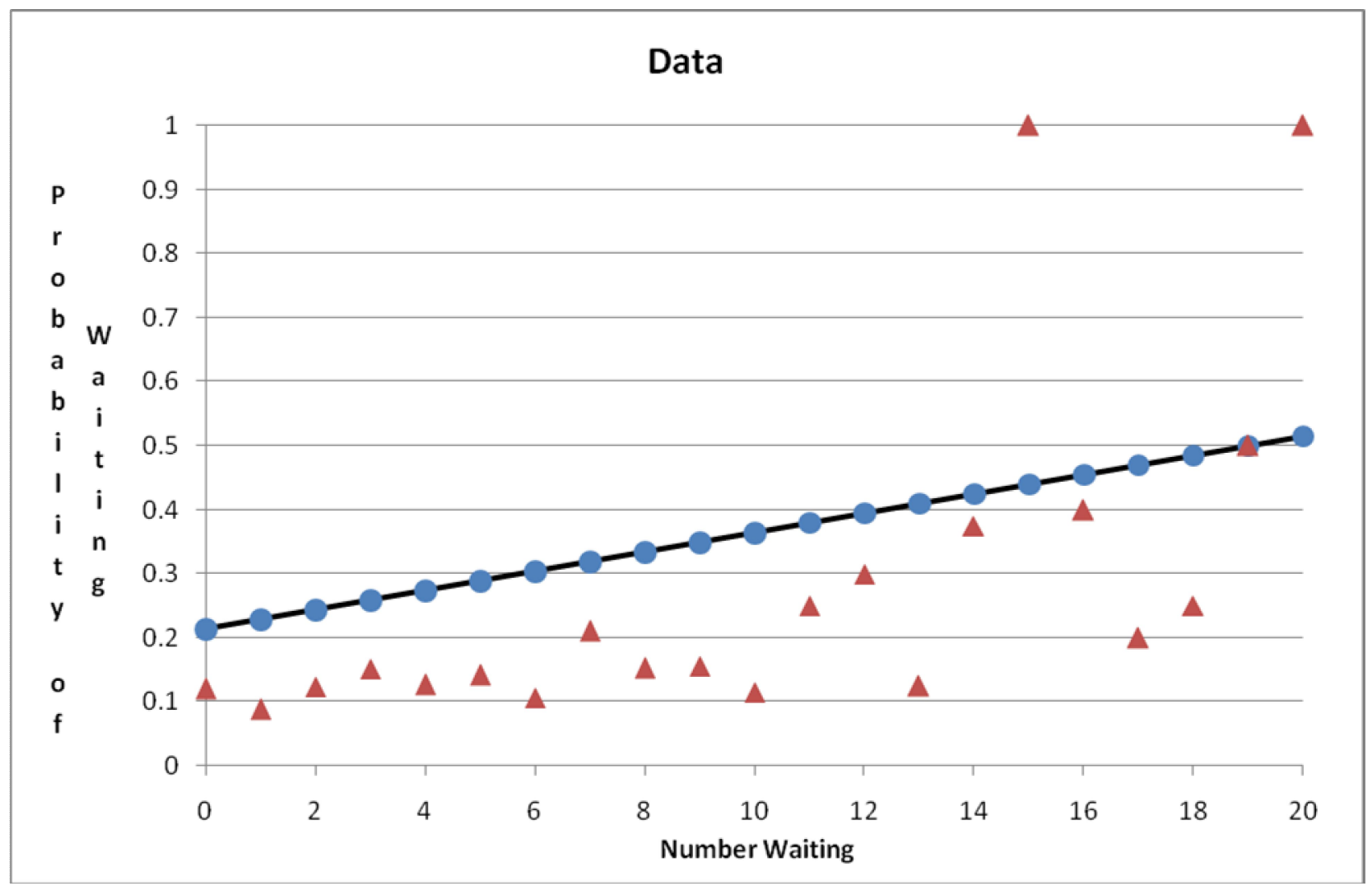

Table 1. Probability of Waiting

\begin{tabular}{|l|c|c|c|}
\hline$m$ & .0150 & .0135 & .0141 \\
& $(.005)$ & $(.005)$ & $(.006)$ \\
\hline$n$ & & .0058 & .0055 \\
& & $(.006)$ & $(.006)$ \\
\hline Temperature & & & -.0006 \\
& & & $(.002)$ \\
\hline Constant & .2142 & .1692 & .2021 \\
& $(.036)$ & $(.060)$ & $(.132)$ \\
\hline $\mathrm{R}^{2}$ & .0309 & .0340 & .0343 \\
& & & \\
\hline
\end{tabular}

Note: 272 observations 


\section{Appendix}

\section{Explanation}

To find the generalized equation, we separated our indifference equation into $S\left(T_{E} / B\right)$ and $T_{W}$. Because $S$ is assumed constant and known, we only needed to examine $T_{W}$. We constructed a diagram to simplify finding $T_{W}$. In the diagram, the probability of any arrival combination, $\frac{1}{n !}$ and non-unique values for the expected time since the last bus arrived (only $(n-$ 1)! of the arrival combinations are unique) have already been factored and canceled out because all travelers are assumed to be the same.

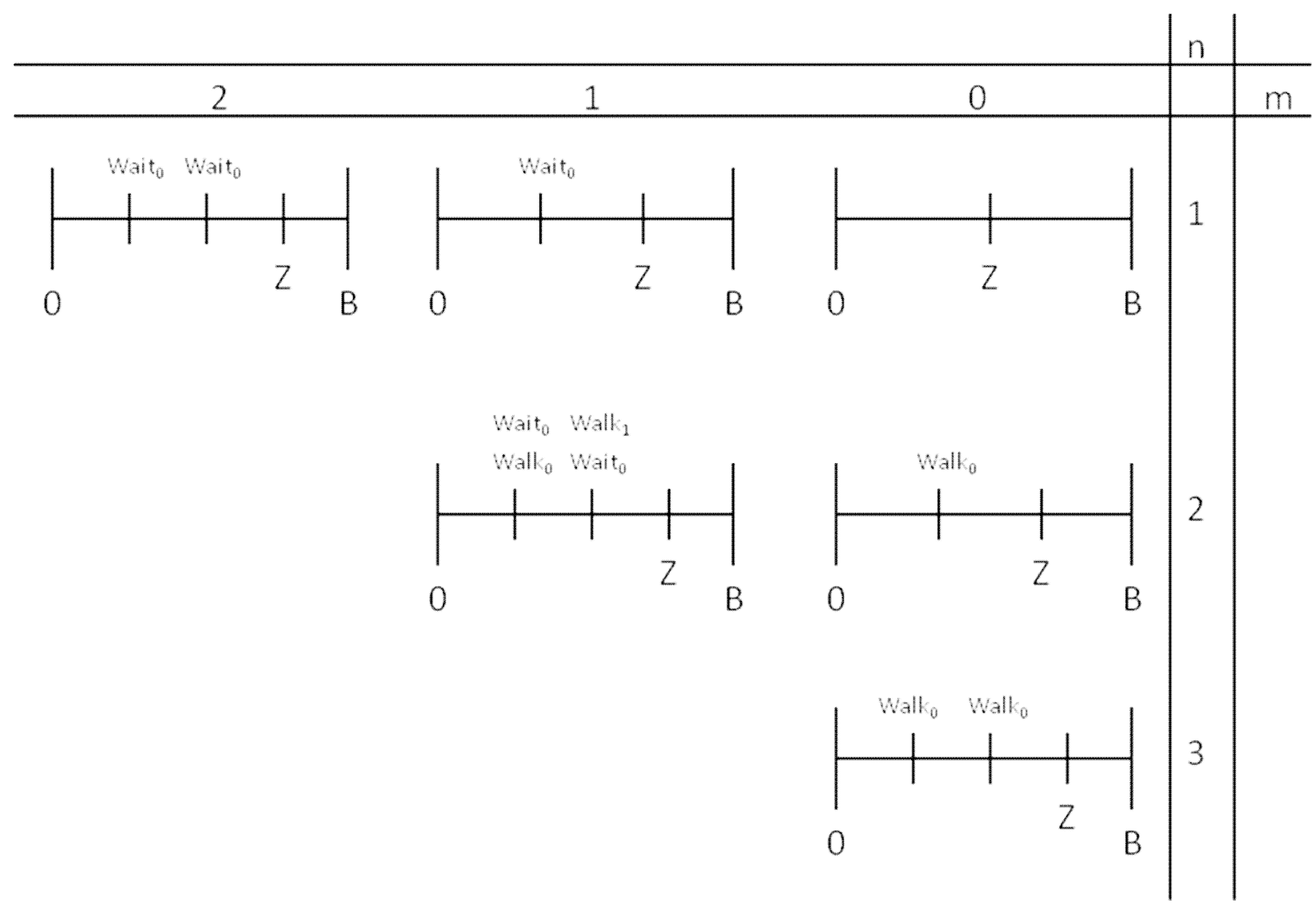




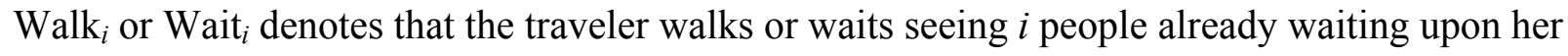
arrival. $n$ still denotes the total number of travelers who will arrive over the entire bus headway $B$ and $m$ still denotes the number of travelers seen waiting

As an example, to use the diagram to find $n=3 m=1$, we first found $n=3$ on the right hand side. We then followed $n=3$ diagonally upwards and leftwards until we reached the correct total number of travelers and number of travelers seen waiting:

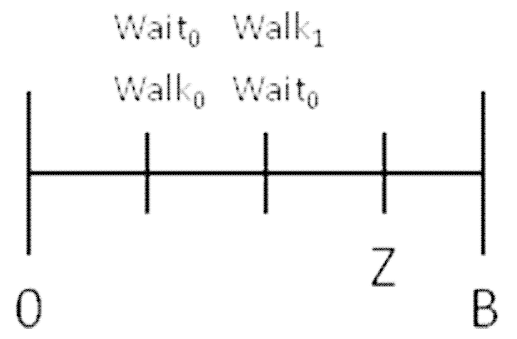

To find the expected wait time, we summed each combination of waits and walks multiplied by the expected wait time, and then divided that by the sum of each combination of waits and walks. The expected times since the last bus left for any $n$ and $m$ is $\frac{(n-m) E}{n+1}, \frac{(n-m-1) B}{n+1}, \ldots, \frac{B}{n+1}$. In this case, we started with $\frac{B}{n+1}=\frac{E}{4}$ :

$$
\frac{\frac{B}{4}\left(1-\sigma_{0}\right)\left(\sigma_{1}\right)+\frac{B}{4}\left(\sigma_{0}\right)\left(1-\sigma_{0}\right)}{\left(1-\sigma_{0}\right)\left(\sigma_{1}\right)+\left(\sigma_{0}\right)\left(1-\sigma_{0}\right)}
$$

We then moved up the column to:

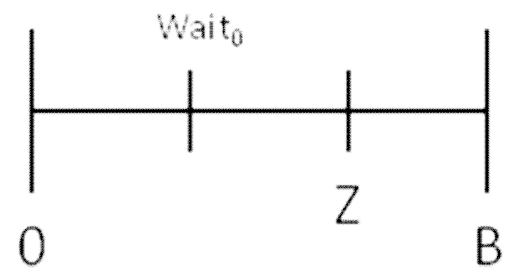

Assuming that a third person arrived after $Z$, we summed the expected wait time for (2) with the expected wait time for (1). The solution for $T_{W}$ if $n=3 m=1$ is: 


$$
\frac{\frac{B}{4}\left(1-\sigma_{0}\right)\left(\sigma_{1}\right)+\frac{B}{4}\left(\sigma_{0}\right)\left(1-\sigma_{0}\right)+\frac{2 B}{4}\left(1-\sigma_{0}\right)}{\left(1-\sigma_{0}\right)\left(\sigma_{1}\right)+\left(\sigma_{0}\right)\left(1-\sigma_{0}\right)+\left(1-\sigma_{0}\right)}
$$

This pattern works for any $B, n, m$, and $\sigma_{i}$. Notice that the probabilities of waiting, $1-\sigma_{i}$, will cancel out because for any $m$, the travelers wait in all possibilities because $Z$ knows how many people are waiting. Therefore, we only needed to include travelers walking in our generalized equation.

The series of probabilities of walking matched the series of expected times since the last bus left with one extra complexity. For all unique combinations of integers $\geq 0$ where $a_{\beta}+$ $b_{\beta}+\ldots+z_{\beta}=\beta$, the generalized series of probabilities that a traveler walks is:

$$
\sigma_{0}^{a_{0}} \sigma_{1}^{b_{0}} \ldots o_{m}^{z_{0}}, \sigma_{0}^{a_{1}} \sigma_{1}^{b_{1}} \ldots o_{m}^{z_{1}}, \ldots, \sigma_{0}^{a_{n-m-2}} \sigma_{1}^{b_{n-m-2}} \ldots o_{m}^{z_{n-m-2}}, \sigma_{0}^{a_{n-m-1}} \sigma_{1}^{b_{n-m-1}} \ldots o_{m}^{z_{n-m-1}}
$$

For example, if $n=3 m=1$, then the probabilities of walking will be:

$$
\begin{gathered}
\sigma_{0}^{a_{0}} \sigma_{1}^{b_{9}} \text { and } \sigma_{0}^{a_{1}} \sigma_{1}^{b_{1}} \\
\sigma_{0}^{0} \sigma_{1}^{0}, \sigma_{0}^{0} \sigma_{1}^{1} \text { and } \sigma_{0}^{1} \sigma_{1}^{0} \\
1, \sigma_{1} \text { and } \sigma_{0}
\end{gathered}
$$

We paired these probabilities of walking with the expected times since the last bus left to find a generalized $T_{W}$. Our generalized $T_{W}$ was placed back into the indifference equation:

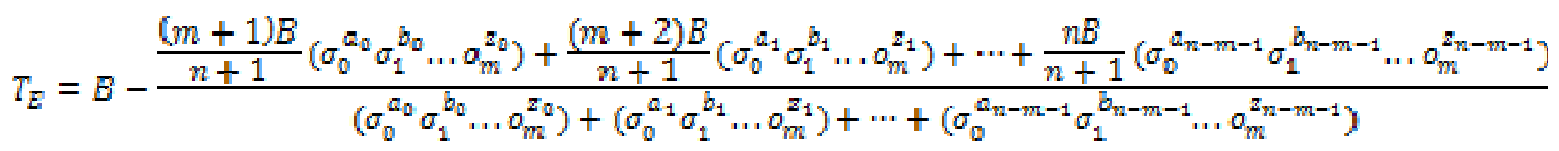

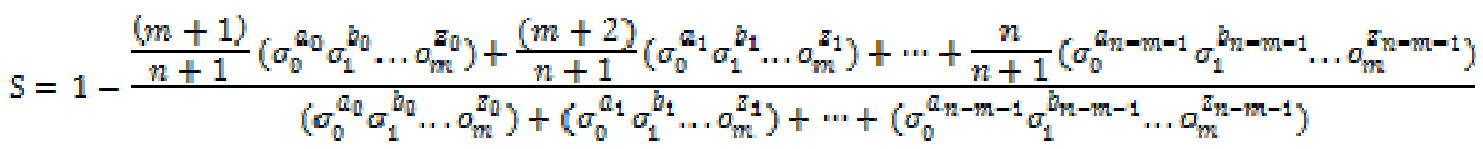




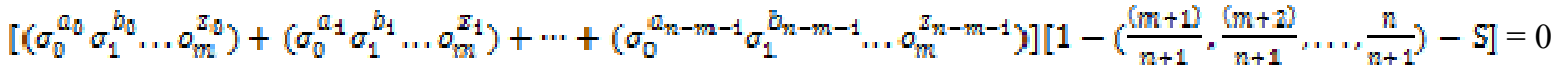

$$
\begin{aligned}
& \sigma_{0}^{a_{a}} \sigma_{1}^{B_{n}} \ldots \sigma_{m}^{x_{0}}\left(\frac{n-m}{n+1}-s\right)+\sigma_{0}^{a_{1}} \sigma_{1}^{b_{1}} \ldots \sigma_{m}^{x_{1}}\left(\frac{n-m-1}{n+1}-s\right)+\cdots \\
& +\sigma_{0}^{a_{n-m-2}} \sigma_{1}^{b_{n-m-2}} \ldots \sigma_{m}^{x_{m-m-2}}\left(\frac{2}{n+1}-s\right)+\sigma_{0}^{a_{m-m-1}} \sigma_{1}^{B_{n-m-1}} \ldots \sigma_{m}^{a_{n-m-1}}\left(\frac{1}{n+1}-s\right)=0
\end{aligned}
$$

For all unique combinations of integers $\geq 0$ where $a_{\beta}+b_{\beta}+\ldots+z_{\beta}=\beta$

\section{Examples}

If $n=3 m=1$ :

$$
\begin{gathered}
\sigma_{0}^{0} \sigma_{1}^{0}\left(\frac{n-m}{n+1}-s\right)+\sigma_{0}^{0} \sigma_{1}^{1}\left(\frac{n-m-1}{n+1}-s\right)+\sigma_{0}^{1} \sigma_{1}^{0}\left(\frac{n-m-1}{n+1}-s\right)=0 \\
(1)\left(\frac{2}{4}-s\right)+\sigma_{1}\left(\frac{1}{4}-s\right)+\sigma_{0}\left(\frac{1}{4}-s\right)=0 \\
\sigma_{1}=\frac{-\sigma_{0}\left(\frac{1}{4}-s\right)-\left(\frac{2}{4}-s\right)}{\left(\frac{1}{4}-s\right)}
\end{gathered}
$$

If $n=3 m=0$ :

$$
\begin{gathered}
\sigma_{0}^{0}\left(\frac{n-m}{n+1}-5\right)+\sigma_{0}^{1}\left(\frac{n-m-1}{n+1}-s\right)+\sigma_{0}^{2}\left(\frac{n-m-2}{n+1}-s\right)=0 \\
(1)\left(\frac{3}{4}-s\right)+\sigma_{0}\left(\frac{2}{4}-s\right)+\sigma_{0}^{2}\left(\frac{1}{4}-s\right)=0 \\
\sigma_{0}^{2}\left(\frac{1}{4}-s\right)+\sigma_{0}\left(\frac{2}{4}-s\right)+\left(\frac{3}{4}-5\right)=0
\end{gathered}
$$

